\title{
Delay-Dependent Stability Analysis of Uncertain Fuzzy Systems with State and Input Delays under Imperfect Premise Matching
}

\author{
Zejian Zhang, ${ }^{1,2}$ Xiao-Zhi Gao, ${ }^{2}$ Kai Zenger, ${ }^{2}$ and Xianlin Huang ${ }^{1}$ \\ ${ }^{1}$ Center for Control Theory and Guidance Technology, Harbin Institute of Technology, Harbin 150001, China \\ ${ }^{2}$ Department of Automation and Systems Technology, Aalto University School of Electrical Engineering, 00076 Aalto, Finland \\ Correspondence should be addressed to Zejian Zhang; zzhanghit@gmail.com
}

Received 1 December 2012; Accepted 4 February 2013

Academic Editor: Peng Shi

Copyright (c) 2013 Zejian Zhang et al. This is an open access article distributed under the Creative Commons Attribution License, which permits unrestricted use, distribution, and reproduction in any medium, provided the original work is properly cited.

\begin{abstract}
This paper discusses the stability and stabilization problem for uncertain T-S fuzzy systems with time-varying state and input delays. A new augmented Lyapunov function with an additional triple-integral term and different membership functions of the fuzzy models and fuzzy controllers are introduced to derive the stability criterion, which is less conservative than the existing results. Moreover, a new flexibility design method is also provided. Some numerical examples are given to demonstrate the effectiveness and less conservativeness of the proposed method.
\end{abstract}

\section{Introduction}

Since the Takagi-Sugeno (T-S) [1] fuzzy systems were firstly proposed in 1985, their stability analysis has received considerable research attention $[2,3]$. However, most of the existing results are only for the T-S fuzzy systems free of time delay. Actually, time delay often occurs in many practical systems, such as $[4,5]$. It has been shown that the existence of time delay usually becomes the source of instability and deteriorated performance of systems. Therefore, study of the time delay is important in both theory and practice [6]. The first stability analysis work on the T-S fuzzy systems with time delay is done in $[7,8]$ by using the Lyapunov-Razumikhin functional approach. If some uncertainties exist in a T-S fuzzy time delay system, they may also significantly affect the system performances and even cause unstable system outputs. Therefore, the issue of the stability for the uncertain T-S fuzzy time delay systems has been widely explored [9-11]. In the literature, two basic approaches have been utilized, that is, delay-independent approach [12] and delay-dependent approach. The latter makes use of the information on the length of the delays, and it is less conservative than the former one. A lot of stability analysis results have been reported based on the delay-dependent approach $[13,14]$.
During the recent years, some research work for different types of the delays of the T-S fuzzy systems has been published, such as constant delay $[15,16]$, bounded time delay [17], time varying delay [18], and interval time varying delay $[19,20]$. However, all these results are only for the TS fuzzy systems with state delays. Thus, they may be invalid when applied to the systems with input delays. As we know, input delays extensively exist in industrial processes and can cause instability or serious performance deterioration. In fact, in modern industrial systems, sensors, controllers, and plants are often connected together, and the sampled data and controller signals are transmitted through networks. In view of this, the input delays should be taken into consideration for robust controller design. Intensive results on the stabilization for the T-S fuzzy systems with state and input delays are reported in [21-28]. For example, the work in [21] is based on the linear systems with input delays. In $[22,24]$, the authors only consider the fuzzy systems with constant input delays, and their results are conservative. In [23], the authors study the fuzzy systems with both the state and input delays. Unfortunately, the results are obtained without any uncertainty, and the state delay is assumed to be equal to the input delay. In [26], the uncertainty has been considered in the analysis, but the state delay is also assumed 
to be the same as the input delay. Some interesting results for the uncertain fuzzy systems with state and input delays have been obtained in $[25,27,28]$, most of which introduce some Lyapunov-Krasovskii functions containing integral terms, for example, $\int_{t-\tau}^{t} x^{T}(s) Q x(s) d s$, and double-integral terms, $\int_{-\tau}^{0} \int_{t+\theta}^{t} \dot{x}^{T}(s) Q \dot{x}(s) d s d \theta$. Several triple-integral terms are used in the Lyapunov function [29] to yield less conservative results for the fuzzy systems with state delay. A large portion of robust controller design topics have been investigated on the basis of the Parallel Distribution Compensation (PDC) design technique, where the fuzzy controller shares the same premise membership functions as those of the T-S fuzzy time delay model [7-11, 15-20, 22-28]. As a matter of fact, if the membership functions in the premise of the fuzzy rules of the fuzzy controllers are allowed to be designed arbitrarily, we can even achieve better design flexibility. For instance, a fuzzy controller not sharing the same premise rules as those of the T-S fuzzy model referred to as imperfect premise matching is employed to control the nonlinear plants [30, 31], and [12] extends the available results to the T-S fuzzy time-delay systems with only the state delay.

In this paper, an augmented Lyapunov-Krasovskii function that contains a triple-integral term is introduced to investigate the stability and stabilization problem for uncertain $\mathrm{T}$ $S$ fuzzy systems with the state and input delays under the imperfect premise matching, in which the fuzzy time delay model and fuzzy controller are with different premise. Some less conservative delay-dependent stability and robust stability conditions are obtained by two integral inequalities and a parameterized model transformation method. Moreover, different from the general PDC design technique, a new design approach of robust stable controllers is proposed. Two simulation examples are further given to illustrate that the proposed design methods are less conservative and more flexible.

\section{System Description and Preliminaries}

Let $r$ be the number of the fuzzy rules describing the time delay nonlinear plant. The $i$ th rule can be represented as follows.

$$
\begin{aligned}
& \text { If } f_{1}(x(t)) \text { is } M_{1}^{i} \text { and } \ldots \text { and } f_{p}(x(t)) \text { is } M_{p}^{i} \\
& \begin{aligned}
\dot{x}(t)= & \left(A_{1 i}+\Delta A_{1 i}(t)\right) x(t)+\left(A_{2 i}+\Delta A_{2 i}(t)\right) \\
& \times x\left(t-d_{1}(t)\right)+\left(B_{i}+\Delta B_{i}(t)\right) u\left(t-d_{2}(t)\right),
\end{aligned} \\
& x(t)=\varphi(t), \quad t \in\left[-\max \left(h_{1}, h_{2}\right), 0\right],
\end{aligned}
$$

where $M_{\alpha}^{i}$ is a fuzzy term of rule $i$ corresponding to the function $f_{\alpha}(x(t)), \alpha=1,2, \ldots, p ; i=1,2, \ldots, r . x(t) \in R^{n}$ is the system state vector, and $u(t) \in R^{m}$ is the input vector. The matrices $A_{1 i}, A_{2 i}$, and $B_{i}, i=1,2, \ldots, r$, are of appropriate dimensions. The initial condition $\varphi(t)$ is a continuous vectorvalued function. The delays $d_{1}(t)$ and $d_{2}(t)$ are time varying, and satisfy

$$
\begin{array}{ll}
0 \leq d_{1}(t) \leq h_{1}, & \dot{d}_{1}(t) \leq \mu_{1}, \\
0 \leq d_{2}(t) \leq h_{2}, & \dot{d}_{2}(t) \leq \mu_{2},
\end{array}
$$

where $h_{i}$ are constants representing the upper bound of the delay. $\mu$ is a positive constant. $\Delta A_{1 i}, \Delta A_{2 i}$, and $\Delta B_{i}$ denote the uncertainties in the system, and they are the form of

$$
\begin{array}{r}
{\left[\begin{array}{lll}
\Delta A_{1 i} & \Delta A_{2 i} & \Delta B_{i}
\end{array}\right]=D_{i} K_{i}(t)\left[\begin{array}{lll}
E_{1 i} & E_{2 i} & E_{b i}
\end{array}\right],} \\
i=1,2, \ldots, r,
\end{array}
$$

where $D_{i}, E_{1 i}, E_{2 i}$, and $E_{b i}$ are known constant matrices of appropriate dimensions, and $K_{i}(t)$ is unknown matrix function satisfying $K_{i}^{T}(t) K_{i}(t) \leq I$. $I$ is an appropriately dimensioned identity matrix. Hence, the overall fuzzy model can be formulized as follows:

$$
\begin{aligned}
\dot{x}(t)=\sum_{i=1}^{r} w_{i}(x(t))[ & \left(A_{1 i}+\Delta A_{1 i}(t)\right) x(t)+\left(A_{2 i}+\Delta A_{2 i}(t)\right) \\
& \times x\left(t-d_{1}(t)\right)+\left(B_{i}+\Delta B_{i}(t)\right) \\
& \left.\times u\left(t-d_{2}(t)\right)\right],
\end{aligned}
$$

where

$$
\begin{array}{r}
\sum_{i=1}^{r} w_{i}(x(t))=1, \quad w_{i}(x(t)) \geq 0, \\
w_{i}(x(t))=\frac{\mu_{i}(x(t))}{\sum_{i=1}^{r} \mu_{i}(x(t))}, \\
\mu_{i}(x(t))=\prod_{\alpha=1}^{p} \mu_{M_{\alpha}^{i}}\left(f_{\alpha}(x(t))\right) .
\end{array}
$$

$w_{i}(x(t))$ is the normalized grade of membership function that is a nonlinear function of $x(t) \cdot \mu_{M_{\alpha}^{i}}\left(f_{\alpha}(x(t))\right)$ is the grade of the membership corresponding to the fuzzy term of $M_{\alpha}^{i}$.

Different from the popular PDC design technique, the following fuzzy control law under imperfect premise matching is employed to deal with the problem of stabilization via state feedback. Under the imperfect premise matching, the $j$ th rule of the fuzzy controller is defined as follows.

$$
\begin{array}{r}
\text { If } g_{1}(x(t)) \text { is } N_{1}^{i} \text { and } \ldots \text { and } g_{q}(x(t)) \text { is } N_{q}^{i} \\
u(t)=F_{j} x(t), \quad j=1,2, \ldots, r,
\end{array}
$$

where $N_{\beta}^{j}$ denotes the fuzzy set. $q$ is a positive integer, and $F_{j} \in R^{m \times n}$ is the feedback gain of rule $j$. The state feedback fuzzy control law is represented by

$$
u(t)=\sum_{j=1}^{r} m_{j}(x(t)) F_{j} x(t),
$$


where

$$
\begin{array}{r}
\sum_{j=1}^{r} m_{j}(x(t))=1, \quad m_{j}(x(t)) \geq 0, \\
m_{j}(x(t))=\frac{v_{j}(x(t))}{\sum_{j=1}^{r} v_{j}(x(t))}, \\
v_{j}(x(t))=\prod_{\beta=1}^{q} v_{N_{\beta}^{j}}\left(g_{\beta}(x(t))\right) .
\end{array}
$$

$m_{j}(x(t))$ is the normalized grade of membership function that is a nonlinear function of $x(t) \cdot v_{N_{\beta}^{j}}\left(g_{\beta}(x(t))\right)$ is the grade of the membership corresponding to the fuzzy term of $N_{\beta}^{j}$.

As the time varying delay is included in the control input, we have

$$
u\left(t-d_{2}(t)\right)=\sum_{j=1}^{r} m_{j}\left(x\left(t-d_{2}(t)\right)\right) F_{j} x\left(t-d_{2}(t)\right),
$$

where

$$
\sum_{j=1}^{r} m_{j}\left(x\left(t-d_{2}(t)\right)\right)=1, \quad m_{j}\left(x\left(t-d_{2}(t)\right)\right) \geq 0 .
$$

Lemma 1 (see [32] (Schur complement)). Given constant matrices $\Omega_{1}, \Omega_{2}$, and $\Omega_{3}$, where $\Omega_{1}=\Omega_{1}^{T}$ and $\Omega_{2}=\Omega_{2}^{T}$, there is $\Omega_{1}+\Omega_{3}^{T} \Omega_{2}^{-1} \Omega_{3}<0$, if and only if

$$
\left[\begin{array}{cc}
\Omega_{1} & \Omega_{3}^{T} \\
* & -\Omega_{2}
\end{array}\right]<0 \quad \text { or } \quad\left[\begin{array}{cc}
-\Omega_{2} & \Omega_{3} \\
* & -\Omega_{1}
\end{array}\right]<0 .
$$

Lemma 2 (see [33]). Let $Q=Q^{T}, D, E$, and $K(t)$ satisfies $K^{T}(t) K(t) \leq I$; the following inequality holds

$$
Q+D K(t) E+E^{T} K^{T}(t) D^{T}<0,
$$

if and only if the following inequality holds for any smaller $\varepsilon>$ 0 :

$$
Q+\varepsilon^{-1} D D^{T}+\varepsilon E^{T} E<0 .
$$

Lemma 3 (see [34]). For any constant matrix $\Sigma=\Sigma^{T}>0$ and a scalar $\tau>0$ such that the following integrations are well defined, we have

$$
\begin{aligned}
& -\int_{t-\tau}^{t} x^{T}(s) \Sigma x(s) d s \\
& \quad \leq-\frac{1}{\tau}\left(\int_{t-\tau}^{t} x(s) d s\right)^{T} \Sigma\left(\int_{t-\tau}^{t} x(s) d s\right), \\
& -\int_{-\tau}^{0} \int_{t+\theta}^{t} x^{T}(s) \Sigma x(s) d s d \theta \\
& \leq-\frac{1}{\tau^{2}}\left(\int_{-\tau}^{0} \int_{t+\theta}^{t} x(s) d s d \theta\right)^{T} \Sigma\left(\int_{-\tau}^{0} \int_{t+\theta}^{t} x(s) d s d \theta\right) .
\end{aligned}
$$

\section{Main Results}

In this section, some new delay-dependent criteria for the T-S fuzzy systems with state and input delays are proposed by introducing a novel Lyapunov function with an additional triple-integral term under the imperfect premise matching. Moreover, a robust stabilization criterion is also investigated.

3.1. Stability of Nominal Fuzzy Systems. Firstly, we consider the control design of a state feedback control law under the imperfect premise matching that stabilizes the following nominal fuzzy time varying delay system:

$$
\begin{aligned}
& \dot{x}(t)=\sum_{i=1}^{r} \sum_{j=1}^{r} w_{i}(x(t)) m_{j}\left(x\left(t-d_{2}(t)\right)\right) \\
& \times {\left[A_{1 i} x(t)+A_{2 i} x\left(t-d_{1}(t)\right)+B_{i} F_{j} x\right.} \\
&\left.\times\left(t-d_{2}(t)\right)\right], \\
& x(t)=\varphi(t), \quad t \in\left[-\max \left(h_{1}, h_{2}\right), 0\right] .
\end{aligned}
$$

Theorem 4. Given scalars $h_{1} \geq 0, h_{2} \geq 0, \mu_{1}, \mu_{2}$, and $t_{i}(i=2, \ldots, 8)$, the closed-loop system (15) is asymptotically stable for any $0 \leq d_{i}(t) \leq h_{i}(i=1,2)$ via the imperfect premise matching controller design technique, if the membership functions of the fuzzy model and fuzzy controller satisfy $m_{j}\left(x\left(t-d_{2}(t)\right)\right)-\rho_{j} w_{j}(x(t)) \geq 0$ for all $j, x(t)$ and $x\left(t-d_{2}(t)\right)$, where $0<\rho_{j}<1$, and there exist matrices $\bar{P}_{11}>0, \bar{P}_{22}>0, \bar{W}_{11}>0, \bar{W}_{22}>0, \bar{Z}_{11}>0, \bar{Z}_{22}>$ $0, \bar{N}_{11}>0, \bar{N}_{22}>0, \bar{Q}_{i}>0, \bar{M}_{i}>0, \bar{R}_{i}>0(i=1,2)$, $\Lambda_{i}=\Lambda_{i}^{T} \in R^{8 n \times 8 n}>0(i=1,2, \ldots, r)$, and

$$
\begin{array}{ll}
\bar{P}=\left[\begin{array}{cc}
\bar{P}_{11} & \bar{P}_{12} \\
* & \bar{P}_{22}
\end{array}\right]>0, & \bar{W}=\left[\begin{array}{cc}
\bar{W}_{11} & \bar{W}_{12} \\
* & \bar{W}_{22}
\end{array}\right]>0, \\
\bar{Z}=\left[\begin{array}{cc}
\bar{Z}_{11} & \bar{Z}_{12} \\
* & \bar{Z}_{22}
\end{array}\right]>0, & \bar{N}=\left[\begin{array}{cc}
\bar{N}_{11} & \bar{N}_{12} \\
* & \bar{N}_{22}
\end{array}\right]>0,
\end{array}
$$

and real matrices $Y_{j}(j=1,2, \ldots, r), \bar{P}_{12} \bar{W}_{12} \bar{Z}_{12} \bar{N}_{12}$, and $X$ such that (30)-(32) hold. In addition, the stabilizing control law is given by

$$
u(t)=\sum_{j=1}^{r} m_{j}\left(x\left(t-d_{2}(t)\right)\right) Y_{j} X^{-T} x(t)
$$

Proof. Choose a fuzzy weighting-dependent LyapunovKrasovskii functional candidate as

$$
V(x(t))=V_{1}(x(t))+V_{2}(x(t))+V_{3}(x(t))+V_{4}(x(t))
$$

$$
+V_{5}(x(t))
$$




$$
\begin{aligned}
V_{1}(x(t))= & \xi_{1}^{T}(t) P \xi_{1}(t)+\xi_{2}^{T}(t) W \xi_{2}(t), \\
V_{2}(x(t))= & \int_{-h_{1}}^{0} \int_{t+\theta}^{t} \xi^{T}(s) Z \xi(s) d s d \theta \\
& +\int_{-h_{2}}^{0} \int_{t+\theta}^{t} \xi^{T}(s) N \xi(s) d s d \theta, \\
V_{3}(x(t))= & \int_{t-d_{1}(t)}^{t} x^{T}(s) Q_{1} x(s) d s \\
& +\int_{t-d_{2}(t)}^{t} x^{T}(s) Q_{2} x(s) d s, \\
V_{4}(x(t))= & \int_{t-h_{1}}^{t} x^{T}(s) M_{1} x(s) d s+\int_{t-h_{2}}^{t} x^{T}(s) M_{2} x(s) d s, \\
V_{5}(x(t))= & \int_{-h_{1}}^{0} \int_{\theta}^{0} \int_{t+\lambda}^{t} \dot{x}^{T}(s) R_{1} \dot{x}(s) d s d \lambda d \theta \\
& +\int_{-h_{2}}^{0} \int_{\theta}^{0} \int_{t+\lambda}^{t} \dot{x}^{T}(s) R_{2} \dot{x}(s) d s d \lambda d \theta,
\end{aligned}
$$

where

$$
\begin{gathered}
\xi_{1}^{T}(t)=\left[\begin{array}{ll}
x^{T}(t) & \left(\int_{t-h_{1}}^{t} x(s) d s\right)^{T}
\end{array}\right], \\
\xi_{2}^{T}(t)=\left[\begin{array}{ll}
x^{T}(t) & \left(\int_{t-h_{2}}^{t} x(s) d s\right)^{T}
\end{array}\right], \\
\xi^{T}(t)=\left[\begin{array}{ll}
x^{T}(t) & \dot{x}^{T}(t)
\end{array}\right], \\
P=\left[\begin{array}{cc}
P_{11} & P_{12} \\
* & P_{22}
\end{array}\right]>0, \quad W=\left[\begin{array}{cc}
W_{11} & W_{12} \\
* & W_{22}
\end{array}\right]>0, \\
Z=\left[\begin{array}{cc}
Z_{11} & Z_{12} \\
* & Z_{22}
\end{array}\right]>0, \quad N=\left[\begin{array}{cc}
N_{11} & N_{12} \\
* & N_{22}
\end{array}\right]>0,
\end{gathered}
$$

and $Q_{i}, M_{i}$, and $R_{i}(i=1,2)$ are the positive-definite matrices to be determined.

The derivatives of $V(x(t))$ along the trajectories of system (15) are as follows:

$$
\begin{aligned}
\dot{V}_{1}(x(t))= & 2 \xi_{1}^{T}(t) P \dot{\xi}_{1}(t)+2 \xi_{2}^{T}(t) W \dot{\xi}_{2}(t) \\
= & 2\left[x^{T}(t)\left(\int_{t-h_{1}}^{t} x(s) d s\right)^{T}\right]\left[\begin{array}{cc}
P_{11} & P_{12} \\
* & P_{22}
\end{array}\right] \\
& \times\left[\begin{array}{c}
\dot{x}(t) \\
x(t)-x\left(t-h_{1}\right)
\end{array}\right] \\
& +2\left[x^{T}(t)\left(\int_{t-h_{2}}^{t} x(s) d s\right)^{T}\right]\left[\begin{array}{cc}
W_{11} & W_{12} \\
* & W_{22}
\end{array}\right] \\
& \times\left[\begin{array}{c}
\dot{x}(t) \\
x(t)-x\left(t-h_{2}\right)
\end{array}\right],
\end{aligned}
$$

$$
\begin{aligned}
& \dot{V}_{2}(x(t))=h_{1}\left[x^{T}(t) \dot{x}^{T}(t)\right]\left[\begin{array}{cc}
Z_{11} & Z_{12} \\
* & Z_{22}
\end{array}\right]\left[\begin{array}{c}
x(t) \\
\dot{x}(t)
\end{array}\right] \\
& -\int_{t-h_{1}}^{t}\left[x^{T}(s) \dot{x}^{T}(s)\right]\left[\begin{array}{cc}
Z_{11} & Z_{12} \\
* & Z_{22}
\end{array}\right]\left[\begin{array}{c}
x(s) \\
\dot{x}(s)
\end{array}\right] d s \\
& +h_{2}\left[\begin{array}{ll}
x^{T}(t) & \dot{x}^{T}(t)
\end{array}\right]\left[\begin{array}{cc}
N_{11} & N_{12} \\
* & N_{22}
\end{array}\right]\left[\begin{array}{c}
x(t) \\
\dot{x}(t)
\end{array}\right] \\
& -\int_{t-h_{2}}^{t}\left[x^{T}(s) \dot{x}^{T}(s)\right]\left[\begin{array}{cc}
N_{11} & N_{12} \\
* & N_{22}
\end{array}\right]\left[\begin{array}{c}
x(s) \\
\dot{x}(s)
\end{array}\right] d s, \\
& \dot{V}_{3}(x(t))=x^{T}(t) Q_{1} x(t)-\left(1-\dot{d}_{1}(t)\right) x^{T}\left(t-d_{1}(t)\right) \\
& \times Q_{1} x\left(t-d_{1}(t)\right)+x^{T}(t) Q_{2} x(t) \\
& -\left(1-\dot{d}_{2}(t)\right) x^{T}\left(t-d_{2}(t)\right) Q_{2} x\left(t-d_{2}(t)\right) \\
& \leq x^{T}(t) Q_{1} x(t)+x^{T}(t) Q_{2} x(t)-\left(1-\mu_{1}\right) \\
& \times x^{T}\left(t-d_{1}(t)\right) Q_{1} x\left(t-d_{1}(t)\right)-\left(1-\mu_{2}\right) \\
& \times x^{T}\left(t-d_{2}(t)\right) Q_{2} x\left(t-d_{2}(t)\right) \text {, } \\
& \dot{V}_{4}(x(t))=x^{T}(t) M_{1} x(t)-x^{T}\left(t-h_{1}\right) M_{1} x\left(t-h_{1}\right) \\
& +x^{T}(t) M_{2} x(t)-x^{T}\left(t-h_{2}\right) M_{2} x\left(t-h_{2}\right), \\
& \dot{V}_{5}(x(t))=\frac{1}{2} h_{1}^{2} \dot{x}^{T}(t) R_{1} \dot{x}(t)-\int_{-h}^{0} \int_{t+\theta}^{t} \dot{x}^{T}(s) \\
& \times R_{1} \dot{x}(s) d s d \theta+\frac{1}{2} h_{2}^{2} \dot{x}^{T}(t) R_{2} \dot{x}(t) \\
& -\int_{-h}^{0} \int_{t+\theta}^{t} \dot{x}^{T}(s) R_{2} \dot{x}(s) d s d \theta .
\end{aligned}
$$

From (15), the following equation can be obtained for any matrices $T_{i}, i=1,2, \ldots, 8$,

$$
\begin{aligned}
& 2 \sum_{i=1}^{r} \sum_{j=1}^{r} w_{i}(x(t)) m_{j}\left(x\left(t-d_{2}(t)\right)\right) \\
& \times\left[x^{T}(t) T_{1}+x^{T}\left(t-d_{1}(t)\right) T_{2}+x^{T}\left(t-h_{1}\right) T_{3}\right. \\
& \quad+\left(\int_{t-h_{1}}^{t} x(s) d s\right)^{T} T_{4}+x^{T}\left(t-d_{2}(t)\right) T_{5}+x^{T} \\
& \left.\quad \times\left(t-h_{2}\right) T_{6}+\left(\int_{t-h_{2}}^{t} x(s) d s\right)^{T} T_{7}+\dot{x}^{T}(t) T_{8}\right] \\
& \times\left[A_{1 i} x(t)-A_{2 i} x\left(t-d_{1}(t)\right)\right. \\
& \left.-B_{i} F_{j} x\left(t-d_{2}(t)\right)-\dot{x}(t)\right]=0 .
\end{aligned}
$$


Mathematical Problems in Engineering

5

By Lemma 3, we have

$$
\begin{aligned}
& -\int_{t-h_{1}}^{t}\left[x^{t}(s) \dot{x}^{T}(s)\right]\left[\begin{array}{cc}
Z_{11} & Z_{12} \\
* & Z_{22}
\end{array}\right]\left[\begin{array}{c}
x(s) \\
\dot{x}(s)
\end{array}\right] d s \\
& \leq-\frac{1}{h_{1}}\left[\left(\int_{t-h_{1}}^{t} x(s) d s\right)^{T} x^{T}(t)-x^{T}\left(t-h_{1}\right)\right] \\
& \times\left[\begin{array}{cc}
Z_{11} & Z_{12} \\
* & Z_{22}
\end{array}\right]\left[\begin{array}{c}
\int_{t-h_{1}}^{t} x(s) d s \\
x(t)-x\left(t-h_{1}\right)
\end{array}\right] \\
& -\int_{t-h_{2}}^{t}\left[x^{T}(s) \dot{x}^{T}(s)\right]\left[\begin{array}{cc}
N_{11} & N_{12} \\
* & N_{22}
\end{array}\right]\left[\begin{array}{c}
x(s) \\
\dot{x}(s)
\end{array}\right] d s \\
& \leq-\frac{1}{h_{2}}\left[\left(\int_{t-h_{2}}^{t} x(s) d s\right)^{T} x^{T}(t)-x^{T}\left(t-h_{1}\right)\right] \\
& \times\left[\begin{array}{cc}
N_{11} & N_{12} \\
* & N_{22}
\end{array}\right]\left[\begin{array}{c}
\int_{t-h_{2}}^{t} x(s) d s \\
x(t)-x\left(t-h_{2}\right)
\end{array}\right] \\
& -\int_{-h_{i}}^{0} \int_{t+\theta}^{t} \dot{x}^{T}(s) R_{i} \dot{x}(s) d s d \theta \\
& \leq-\frac{2}{h_{i}^{2}}\left(\int_{-h_{i}}^{0} \int_{t+\theta}^{t} \dot{x}(s) d s d \theta\right)^{T} R_{i}\left(\int_{-h_{i}}^{0} \int_{t+\theta}^{t} \dot{x}(s) d s d \theta\right) \\
& =-\frac{2}{h_{i}^{2}}\left(h_{i} x(t)-\int_{t-h_{i}}^{t} x(s) d s\right)^{T} \\
& \times R_{i}\left(h_{i} x(t)-\int_{t-h_{i}}^{t} x(s) d s\right) \quad i=1,2 .
\end{aligned}
$$

Using the above inequalities, and with the zero quantities (21), we can obtain

$$
\begin{aligned}
& \dot{V}\left(x_{t}\right) \\
& \leq 2\left[x^{T}(t)\left(\int_{t-h_{1}}^{t} x(s) d s\right)^{T}\right]\left[\begin{array}{cc}
P_{11} & P_{12} \\
* & P_{22}
\end{array}\right] \\
& \times\left[\begin{array}{c}
\dot{x}(t) \\
x(t)-x\left(t-h_{1}\right)
\end{array}\right]+h_{1}\left[\begin{array}{ll}
x^{T}(t) & \dot{x}^{T}(t)
\end{array}\right] \\
& \times\left[\begin{array}{cc}
Z_{11} & Z_{12} \\
* & Z_{22}
\end{array}\right]\left[\begin{array}{c}
x(t) \\
\dot{x}(t)
\end{array}\right]+h_{2}\left[x^{T}(t) \dot{x}^{T}(t)\right]\left[\begin{array}{cc}
N_{11} & N_{12} \\
* & N_{22}
\end{array}\right]
\end{aligned}
$$

$$
\begin{aligned}
& \times\left[\begin{array}{c}
x(t) \\
\dot{x}(t)
\end{array}\right]-\frac{1}{h_{1}}\left[\left(\int_{t-h_{1}}^{t} x(s) d s\right)^{T} x^{T}(t)-x^{T}\left(t-h_{1}\right)\right] \\
& \times\left[\begin{array}{cc}
Z_{11} & Z_{12} \\
* & Z_{22}
\end{array}\right]\left[\begin{array}{c}
\int_{t-h_{1}}^{t} x(s) d s \\
x(t)-x\left(t-h_{1}\right)
\end{array}\right] \\
& -\frac{1}{h_{2}}\left[\left(\int_{t-h_{2}}^{t} x(s) d s\right)^{T} x^{T}(t)-x^{T}\left(t-h_{2}\right)\right] \\
& \times\left[\begin{array}{cc}
N_{11} & N_{12} \\
* & N_{22}
\end{array}\right]\left[\begin{array}{c}
\int_{t-h_{2}}^{t} x(s) d s \\
x(t)-x\left(t-h_{2}\right)
\end{array}\right]+x^{T}(t) Q_{1} x(t) \\
& +x^{T}(t) Q_{2} x(t)-\left(1-\mu_{1}\right) x^{T}\left(t-d_{1}(t)\right) Q_{1} x \\
& \times\left(t-d_{1}(t)\right)-\left(1-\mu_{2}\right) x^{T}\left(t-d_{2}(t)\right) Q_{2} x \\
& \times\left(t-d_{2}(t)\right)+x^{T}(t) M_{1} x(t)-x^{T}\left(t-h_{1}\right) M_{1} x \\
& \times\left(t-h_{1}\right)+x^{T}(t) M_{2} x(t)-x^{T}\left(t-h_{2}\right) M_{2} x \\
& \times\left(t-h_{2}\right)+\frac{1}{2} h_{1}^{2} \dot{x}^{T}(t) R_{1} \dot{x}(t)-\frac{2}{h_{1}^{2}} \\
& \times\left(h_{1} x(t)-\int_{t-h_{1}}^{t} x(s) d s\right)^{T} R_{1}\left(h_{1} x(t)-\int_{t-h_{1}}^{t} x(s) d s\right) \\
& +\frac{1}{2} h_{2}{ }^{2} \dot{x}^{T}(t) R_{2} \dot{x}(t)-\frac{2}{h_{2}^{2}}\left(h_{2} x(t)-\int_{t-h_{2}}^{t} x(s) d s\right)^{T} \\
& \times R_{2}\left(h_{2} x(t)-\int_{t-h_{2}}^{t} x(s) d s\right) \\
& +2\left[x^{T}(t)\left(\int_{t-h_{2}}^{t} x(s) d s\right)^{T}\right] \\
& \times\left[\begin{array}{cc}
W_{11} & W_{12} \\
* & W_{22}
\end{array}\right]\left[\begin{array}{c}
\dot{x}(t) \\
x(t)-x\left(t-h_{2}\right)
\end{array}\right] \\
& +2 \sum_{i=1}^{r} \sum_{j=1}^{r} w_{i}(x(t)) m_{j}\left(x\left(t-d_{2}(t)\right)\right) \\
& \times\left[x^{T}(t) T_{1}+x^{T}\left(t-d_{1}(t)\right) T_{2}+x^{T}\left(t-h_{1}\right) T_{3}\right. \\
& +\left(\int_{t-h_{1}}^{t} x(s) d s\right)^{T} T_{4}+x^{T}\left(t-d_{2}(t)\right) T_{5}+x^{T} \\
& \left.\times\left(t-h_{2}\right) T_{6}+\left(\int_{t-h_{2}}^{t} x(s) d s\right)^{T} T_{7}+\dot{x}^{T}(t) T_{8}\right] \\
& \times\left[A_{1 i} x(t)-A_{2 i} x\left(t-d_{1}(t)\right)\right. \\
& \left.-B_{i} F_{j} x\left(t-d_{2}(t)\right)-\dot{x}(t)\right] \\
& =\zeta^{T}(t)\left(\sum_{i=1}^{r} \sum_{j=1}^{r} w_{i}(x(t)) m_{j}\left(x\left(t-d_{2}(t)\right)\right) \Phi_{i j}\right) \zeta(t),
\end{aligned}
$$


where

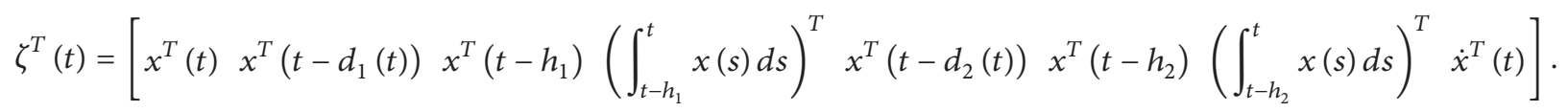

$\Phi_{i j}=$

$\left[\begin{array}{cccccccc}\Phi_{11 i} & \Phi_{12 i} & \Phi_{13} & \Phi_{14} & \Phi_{15 i j} & \Phi_{16} & \Phi_{17} & \Phi_{18} \\ * & \Phi_{22 i} & A_{22}^{T} T_{3}^{T} & A_{21}^{T} T_{4}^{T} & \Phi_{25 i j} & A_{2 i}^{T} T_{6}^{T} & A_{2 i}^{T} T_{7}^{T} & \Phi_{28 i} \\ * & * & \Phi_{33 i} & -P_{22} & T_{3} B_{B} F_{j} & 0 & 0 & -T_{3} \\ * & * & * & \Phi_{44} & T_{4} B_{i} F_{j} & 0 & 0 & 0 \\ * & * & * & * & \Phi_{55 i j} & F_{j}^{T} B_{i}^{T} T_{6}^{T} & F_{j}^{T} B_{i}^{T} T_{7}^{T} & \Phi_{48} \\ * & * & * & * & * & \Phi_{58 i} \\ * & * & * & * & * & * & \Phi_{67} & -T_{6} \\ * & * & * & * & * & * & * & \Phi_{78}\end{array}\right]$,

$\Phi_{11 i}=P_{12}+P_{12}^{T}+W_{12}+W_{12}^{T}+h_{1} Z_{11}+h_{2} N_{11}$

$-\frac{1}{h_{1}} Z_{22}-\frac{1}{h_{2}} N_{22}+Q_{1}+Q_{2}+M_{1}+M_{2}$

$-2 R_{1}-2 R_{2}+T_{1} A_{1 i}+A_{1 i}^{T} T_{1}^{T}$,

$\Phi_{12 i}=T_{1} A_{2 i}+A_{1 i}^{T} T_{2}^{T}$,

$\Phi_{13}=-P_{12}-\frac{1}{h_{1}} Z_{22}+A_{1 i}^{T} T_{3}^{T}$,

$\Phi_{14}=P_{22}-\frac{1}{h_{2}} Z_{12}^{T}+\frac{1}{h_{1}} R_{1}+A_{1 i}^{T} T_{4}^{T}$,

$\Phi_{15 i j}=T_{1} B_{i} F_{j}+A_{1 i}^{T} T_{5}^{T}$,

$\Phi_{16}=-W_{12}-\frac{1}{h_{2}} N_{22}+A_{1 i}^{T} T_{6}^{T}$,

$\Phi_{17}=W_{22}-\frac{1}{h_{2}} N_{12}^{T}+\frac{2}{h_{2}^{2}} R_{2}+A_{1 i}^{T} T_{7}^{T}$,

$\Phi_{18}=P_{11}+W_{11}+h_{1} Z_{12}+h_{2} N_{12}-T_{1}+A_{1 i}^{T} T_{8}^{T}$,

$\Phi_{22 i}=-\left(1-\mu_{1}\right) Q_{1}+T_{2} A_{2 i}+A_{2 i}^{T} T_{2}^{T}$,

$\Phi_{25 i j}=T_{2} B_{i} F_{j}+A_{2 i}^{T} T_{5}^{T}$,

$\Phi_{28 i}=-T_{2}+A_{2 i}^{T} T_{8}^{T}$,

$\Phi_{33}=-\frac{1}{h} Z_{22}-M_{1}$,

$\Phi_{44}=-\frac{1}{h_{1}} Z_{11}-\frac{2}{h_{1}^{2}} R_{1}$,

$\Phi_{48}=P_{12}^{T}-T_{4}$,

$$
\Phi_{55 i}=-\left(1-\mu_{2}\right) Q_{2}+T_{5} B_{i} F_{j}+F_{j}^{T} B_{i}^{T} T_{5}^{T},
$$$$
\Phi_{58 i}=-T_{5}+F_{j}^{T} B_{i}^{T} T_{8}^{T},
$$$$
\Phi_{66}=-\frac{1}{h_{2}} N_{22}-M_{2} \text {, }
$$$$
\Phi_{67}=-\frac{1}{h_{2}} N_{12}^{T}-W_{22}
$$$$
\Phi_{77}=-\frac{1}{h_{2}} N_{11}-\frac{2}{h_{2}^{2}} R_{2},
$$$$
\Phi_{78}=W_{12}^{T}-T_{7},
$$

$$
\Phi_{88}=h_{1} Z_{22}+h_{2} N_{22}+\frac{1}{2} h_{1}^{2} R_{1}+\frac{1}{2} h_{2}^{2} R_{2}-T_{8} .
$$

From (25), it is obvious that if

$$
\sum_{i=1}^{r} \sum_{j=1}^{r} w_{i}(x(t)) m_{j}\left(x\left(t-d_{2}(t)\right)\right) \Phi_{i j}<0
$$

$\dot{V}(x(t))<0$.

From (26), we can discover that the feedback gains are predefined. The following proof presents the controller design method. We first pre- and postmultiply both the

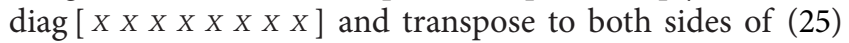
and pre- and postmultiply the $\operatorname{diag}[X X]$ transpose to both sides of $P, W, Z$, and $N$ and the $X$ and transpose to both sides of $Q_{i}, M_{i}$, and $R_{i}, i=1,2$. Let $T_{i}=t_{i} T_{1}(i=2, \ldots, 8)$, and denote new variables $X=T_{1}^{-1}, \bar{R}_{1}=X R_{1} X^{T}, \bar{Q}_{1}=$ $X Q_{1} X^{T}, \bar{M}_{1}=X M_{1} X^{T}, \bar{R}_{2}=X R_{2} X^{T}, \bar{Q}_{2}=X Q_{2} X^{T}, \bar{M}_{2}=$ $X M_{2} X^{T}, \bar{\Lambda}_{i}=X \Lambda_{i} X^{T}, \bar{\Lambda}_{j}=X \Lambda_{j} X^{T}, i=1,2, \ldots, r, \bar{P}_{11}=$ $X P_{11} X^{T}, \bar{P}_{12}=X P_{12} X^{T}, \bar{P}_{22}=X P_{22} X^{T}, \bar{W}_{11}=X W_{11} X^{T}$, $\bar{W}_{12}=X W_{12} X^{T}, \bar{W}_{22}=X W_{22} X^{T}, \bar{Z}_{11}=X Z_{11} X^{T}, \bar{Z}_{12}=$ $X Z_{12} X^{T}, \bar{Z}_{22}=X Z_{22} X^{T}, \bar{N}_{11}=X N_{11} X^{T}, \bar{N}_{12}=X N_{12} X^{T}$, and $\bar{N}_{22}=X N_{22} X^{T}$. With $F_{i}=Y_{i} X^{-T}, i=1,2 \ldots, r$, we next get

$$
\sum_{i=1}^{r} \sum_{j=1}^{r} w_{i}(x(t)) m_{j}\left(x\left(t-d_{2}(t)\right)\right) \bar{\Phi}_{i j}<0
$$


where

$$
\begin{aligned}
& \bar{\Phi}_{i j}= \\
& {\left[\begin{array}{cccccccc}
\bar{\Phi}_{11 i} & \bar{\Phi}_{12 i} & \bar{\Phi}_{13} & \bar{\Phi}_{14} & \bar{\Phi}_{15 i j} & \bar{\Phi}_{16} & \bar{\Phi}_{17} & \bar{\Phi}_{18} \\
* & \bar{\Phi}_{22 i} & t_{3} X A_{2 i}^{T} & t_{4} X A_{2 i}^{T} & \bar{\Phi}_{25 i j} & t_{6} X A_{2 i}^{T} & t_{7} X A_{2 i}^{T} & \bar{\Phi}_{28 i} \\
* & * & \bar{\Phi}_{33 i} & -\bar{P}_{22} & t_{3} B_{i} Y_{j} & 0 & 0 & -t_{3} X^{T} \\
* & * & * & \bar{\Phi}_{44} & t_{4} B_{i} Y_{j} & 0 & 0 & \bar{\Phi}_{48} \\
* & * & * & * & \Phi_{55 i j} & t_{6} Y_{j}^{T} B_{i}^{T} & t_{7} Y_{j}^{T} B_{i}^{T} & \bar{\Phi}_{58 i} \\
* & * & * & * & * & \bar{\Phi}_{66} & \bar{\Phi}_{67} & -t_{6} X^{T} \\
* & * & * & * & * & * & \bar{\Phi}_{77} & \bar{\Phi}_{78} \\
* & * & * & * & * & * & * & \bar{\Phi}_{88}
\end{array}\right],} \\
& \bar{\Phi}_{11 i}=\bar{P}_{12}+\bar{P}_{12}^{T}+\bar{W}_{12}+\bar{W}_{12}^{T}+h_{1} \bar{Z}_{11} \\
& +h_{2} \bar{N}_{11}-\frac{1}{h_{1}} \bar{Z}_{22}-\frac{1}{h_{2}} \bar{N}_{22}+\bar{Q}_{1}+\bar{Q}_{2} \\
& +\bar{M}_{1}+\bar{M}_{2}-2 \bar{R}_{1}-2 \bar{R}_{2}+A_{1 i} X^{T}+X A_{1 i}^{T} \text {, } \\
& \bar{\Phi}_{12 i}=A_{2 i} X^{T}+t_{2} X A_{1 i}^{T} \text {, } \\
& \bar{\Phi}_{13}=-\bar{P}_{12}-\frac{1}{h} \bar{Z}_{22}+t_{3} X A_{1 i}^{T} \\
& \bar{\Phi}_{14}=\bar{P}_{22}-\frac{1}{h_{1}} \bar{Z}_{12}^{T}+\frac{1}{h_{1}} \bar{R}_{1}+t_{4} X A_{1 i}^{T} \text {, } \\
& \bar{\Phi}_{15 i j}=B_{i} Y_{j}+t_{5} X A_{1 i}^{T} \text {, } \\
& \bar{\Phi}_{16}=-\bar{W}_{12}-\frac{1}{h_{2}} \bar{N}_{22}+t_{6} X A_{1 i}^{T} \text {, } \\
& \bar{\Phi}_{17}=\bar{W}_{22}-\frac{1}{h_{2}} \bar{N}_{12}^{T}+\frac{2}{h_{2}^{2}} \bar{R}_{2}+t_{7} X A_{1 i}^{T} \text {, } \\
& \bar{\Phi}_{18}=\bar{P}_{11}+\bar{W}_{11}+h_{1} \bar{Z}_{12}+h_{2} \bar{N}_{12}-X^{T}+t_{8} X A_{1 i}^{T} \text {, } \\
& \bar{\Phi}_{22 i}=-\left(1-\mu_{1}\right) \bar{Q}_{1}+t_{2} A_{2 i} X^{T}+t_{2} X A_{2 i}^{T} \text {, } \\
& \bar{\Phi}_{25 i j}=t_{2} B_{i} Y_{j}+t_{5} X A_{2 i}^{T} \text {, } \\
& \Phi_{28 i}=-t_{2} X^{T}+t_{8} X A_{2 i}^{T} \text {, } \\
& \bar{\Phi}_{33}=-\frac{1}{h_{1}} \bar{Z}_{22}-\bar{M}_{1}, \\
& \bar{\Phi}_{44}=-\frac{1}{h_{1}} \bar{Z}_{11}-\frac{2}{h_{1}^{2}} \bar{R}_{1} \text {, } \\
& \bar{\Phi}_{48}=\bar{P}_{12}^{T}-t_{4} X^{T}, \\
& \bar{\Phi}_{55 i}=-\left(1-\mu_{2}\right) \bar{Q}_{2}+t_{5} B_{i} Y_{j}+t_{5} Y_{j}^{T} B_{i}^{T} \text {, } \\
& \bar{\Phi}_{58 i}=-t_{5} X^{T}+t_{8} Y_{j}^{T} B_{i}^{T}, \\
& \bar{\Phi}_{66}=-\frac{1}{h_{2}} \bar{N}_{22}-\bar{M}_{2} \text {, } \\
& \bar{\Phi}_{67}=-\frac{1}{h_{2}} \bar{N}_{12}^{T}-\bar{W}_{22} \text {, }
\end{aligned}
$$

$$
\begin{gathered}
\bar{\Phi}_{77}=-\frac{1}{h_{2}} \bar{N}_{11}-\frac{2}{h_{2}^{2}} \bar{R}_{2}, \\
\bar{\Phi}_{78}=\bar{W}_{12}^{T}-t_{7} X^{T}, \\
\bar{\Phi}_{88}=h_{1} \bar{Z}_{22}+h_{2} \bar{N}_{22}+\frac{1}{2} h_{1}^{2} \bar{R}_{1}+\frac{1}{2} h_{2}^{2} \bar{R}_{2}-t_{8} X^{T} .
\end{gathered}
$$

Here, “*” denotes the transpose elements in the symmetric positions.

If (27) holds, then $\dot{V}(x(t))<0$. Consider $\sum_{i=1}^{r} \sum_{j=1}^{r} w_{i}(x(t))\left(w_{j}(x(t))-m_{j}\left(x\left(t-d_{2}(t)\right)\right)\right) \Lambda_{i}=0$, where $\Lambda_{i}=\Lambda_{i}^{T} \in R^{8 n \times 8 n}>0, i=1,2, \ldots, r$, are arbitrary matrices. These terms are introduced to (27) to alleviate the conservativeness. From (27), we also have

$$
\begin{aligned}
\bar{\Phi}= & \sum_{i=1}^{r} \sum_{j=1}^{r} w_{i}(x(t)) m_{j}\left(x\left(t-d_{2}(t)\right)\right) \bar{\Phi}_{i j} \\
= & \sum_{i=1}^{r} \sum_{j=1}^{r} w_{i}(x(t))\left(w_{j}(x(t))-m_{j}\left(x\left(t-d_{2}(t)\right)\right)\right. \\
& \left.+\rho_{j} w_{j}(x(t))-\rho_{j} w_{j}(x(t))\right) \Lambda_{i} \\
& +\sum_{i=1}^{r} \sum_{j=1}^{r} w_{i}(x(t)) m_{j}\left(x\left(t-d_{2}(t)\right)\right) \bar{\Phi}_{i j} \\
= & \sum_{i=1}^{r} \sum_{j=1}^{r} w_{i}(x(t)) w_{j}(x(t))\left(\rho_{j} \Phi_{i j}-\rho_{j} \Lambda_{i}+\Lambda_{i}\right) \\
& +\sum_{i=1}^{r} \sum_{j=1}^{r} w_{i}(x(t))\left(m_{j}\left(x\left(t-d_{2}(t)\right)\right)-\rho_{j} w_{j}(x(t))\right) \\
& \times\left(\bar{\Phi}_{i j}-\Lambda_{i}\right) \\
& +\sum_{i=1}^{r} \sum_{i<j}^{r} w_{i} w_{j}\left(\rho_{j} \bar{\Phi}_{i j}+\rho_{i} \bar{\Phi}_{j i}-\rho_{j} \Lambda_{i}-\rho_{i} \Lambda_{j}\right. \\
\leq & \left.\sum_{i=1}^{2} w_{i} \bar{\Phi}_{i i}-\rho_{i} \Lambda_{i}+\Lambda_{i}\right) \\
& +\sum_{i=1}^{r} \sum_{j=1}^{r} w_{i}\left(m_{j}-\rho_{j} w_{j}\right)\left(\bar{\Phi}_{i j}-\Lambda_{i}\right)
\end{aligned}
$$

with $m_{j}\left(x\left(t-d_{2}(t)\right)\right)-\rho_{j} w_{j}(x(t)) \geq 0$ for all $j, x(t)$ and $x(t-$ $\left.d_{2}(t)\right)$. Let

$$
\begin{gathered}
\bar{\Phi}_{i j}-\Lambda_{i}<0, \\
\rho_{i} \bar{\Phi}_{i i}-\rho_{i} \Lambda_{i}+\Lambda_{i}<0, \\
\rho_{j} \bar{\Phi}_{i j}+\rho_{i} \bar{\Phi}_{j i}-\rho_{j} \Lambda_{i}-\rho_{i} \Lambda_{j}+\Lambda_{i}+\Lambda_{j} \leq 0, \quad i<j,
\end{gathered}
$$

for all $i, j=1,2, \ldots, r$. 
Since $\dot{V}(x(t))<0$, the fuzzy control system with the time varying state and input delays (15) is asymptotically stable with the state feedback control law (17).

3.2. Robust Stability of Uncertain Fuzzy Systems. We also examine the design of a robust stable controller for the uncertain system (4) under the imperfect premise matching. Consider the following uncertain fuzzy time varying delay control system:

$$
\begin{aligned}
& \dot{x}(t)=\sum_{i=1}^{r} \sum_{j=1}^{r} w_{i}(x(t)) m_{j}\left(x\left(t-d_{2}(t)\right)\right) \\
& \times {\left[\left(A_{1 i}+\Delta A_{1 i}(t)\right) x(t)+\left(A_{2 i}+\Delta A_{2 i}(t)\right)\right.} \\
& \times x\left(t-d_{1}(t)\right)+\left(B_{i}+\Delta B_{i}(t)\right) \\
&\left.\times u\left(t-d_{2}(t)\right)\right], \\
& x(t)=\varphi(t), \quad t \in {\left[-\max \left(h_{1}, h_{2}\right), 0\right] . }
\end{aligned}
$$

Based on the above results of Theorem 4, a robust stabilization criterion for the T-S fuzzy systems with time varying state and input delays is investigated. The following result can be obtained.

Theorem 5. Given scalars $h_{1} \geq 0, h_{2} \geq 0, \mu_{1}, \mu_{2}$, and $t_{i}(i=$ $2, \ldots, 8)$, the uncertain fuzzy control systems with the time varying state and input delays (33) is robustly stable for any $0 \leq d_{i}(t) \leq h_{i}(i=1,2)$ via the imperfect premise matching controller design technique, if the membership functions of the fuzzy model and fuzzy controller satisfy $m_{j}\left(x\left(t-d_{2}(t)\right)\right)-$ $\rho_{j} w_{j}(x(t)) \geq 0$ for all $j, x(t)$, and $x\left(t-d_{2}(t)\right)$, where $0<$ $\rho_{j}<1$, and there exist common matrices $\bar{P}_{11}>0, \bar{P}_{22}>0$, $\bar{W}_{11}>0, \bar{W}_{22}>0, \bar{Z}_{11}>0, \bar{Z}_{22}>0, \bar{N}_{11}>0, \bar{N}_{22}>0$, $\bar{Q}_{i}>0, \bar{M}_{i}>0, \bar{R}_{i}>0(i=1,2)$ and $\Lambda_{i}=\Lambda_{i}^{T} \in R^{8 n \times 8 n}>$

$0(i=1,2, \ldots, r), \bar{P}>0, \bar{W}>0, \bar{Z}>0, \bar{N}>0$ and some matrices $Y_{j}(j=1,2, \ldots, r), X$ and scalars $\varepsilon_{1 i}>0, \varepsilon_{2 i}>0$, $\varepsilon_{b i j}>0$ satisfy the following LMIs:

$$
\begin{gathered}
\Psi_{i j}-\bar{\Lambda}_{i}<0, \\
\rho_{i} \Psi_{i i}-\rho_{i} \bar{\Lambda}_{i}+\bar{\Lambda}_{i}<0, \\
\rho_{j} \Psi_{i j}+\rho_{i} \Psi_{j i}-\rho_{j} \bar{\Lambda}_{i}-\rho_{i} \bar{\Lambda}_{j}+\bar{\Lambda}_{i}+\bar{\Lambda}_{j}, \leq 0 \quad i<j,
\end{gathered}
$$

where $\Psi_{i j}$ is defined as in (44). The state feedback gains can be constructed as $F_{i}=Y_{i} X^{-T}$.

Proof. If $A_{1 i}, A_{2 i}$, and $B_{i}$ in $\bar{\Phi}_{i j}$ are replaced with $A_{1 i}+$ $D_{i} K_{i}(t) E_{1 i}, A_{2 i}+D_{i} K_{i}(t) E_{2 i}$, and $B_{i}+D_{i} K_{i}(t) E_{b i}$ in (28) of Theorem 4, respectively, (27) for system (33) can be rewritten as

$$
\sum_{i=1}^{r} \sum_{j=1}^{r} w_{i}(x(t)) m_{j}(x(t-d(t))) \Omega_{i j}<0,
$$

where

$$
\begin{gathered}
\Omega_{i j}=\bar{\Phi}_{i j}+\widehat{D}_{1 i}^{T} K_{i}(t) \widehat{E}_{i}+\widehat{E}_{i}^{T} K_{i}^{T}(t) \widehat{D}_{1 i}+\widehat{D}_{2 i}^{T} K_{i}(t) \widehat{E}_{2 i j} \\
+\widehat{E}_{2 i j}^{T} K_{i}^{T}(t) \widehat{D}_{2 i}+\widehat{D}_{b i}^{T} K_{i}(t) \widehat{E}_{b i j}+\widehat{E}_{b i j}^{T} K_{i}^{T}(t) \widehat{D}_{b i}, \\
\widehat{D}_{1 i}=\left[\begin{array}{llllllll}
D_{i}^{T} & 0 & 0 & 0 & 0 & 0 & 0 & 0
\end{array}\right], \\
\widehat{D}_{2 i}=\left[\begin{array}{llllllll}
0 & D_{i}^{T} & 0 & 0 & 0 & 0 & 0 & 0
\end{array}\right], \\
\widehat{D}_{b i}=\left[\begin{array}{llllllll}
0 & 0 & 0 & 0 & D_{i}^{T} & 0 & 0 & 0
\end{array}\right],
\end{gathered}
$$

If $\Omega_{i j}<0$, (37) holds. According to Lemma 2, it is straightforward to know that $\Omega_{i j}<0$ is true, if for each $i$, $j$, there exists scalars $\varepsilon_{1 i}>0, \varepsilon_{2 i}>0$, and $\varepsilon_{b i j}>0$ such that the following inequality holds:

$$
\begin{aligned}
\bar{\Phi}_{i j} & +\varepsilon_{1 i} \widehat{D}_{1 i}^{T} \widehat{D}_{1 i}+\varepsilon_{1 i}^{-1} \widehat{E}_{1 i}^{T} \widehat{E}_{1 i}+\varepsilon_{2 i} \widehat{D}_{2 i}^{T} \widehat{D}_{2 i} \\
& +\varepsilon_{2 i}^{-1} \widehat{E}_{2 i}^{T} \widehat{E}_{2 i}+\varepsilon_{b i j} \widehat{D}_{b i}^{T} \widehat{D}_{b i}+\varepsilon_{b i j}^{-1} \widehat{E}_{b i j}^{T} \widehat{E}_{b i j}<0 .
\end{aligned}
$$

Based on Schur complement, (40) is equivalent to the following inequality:

$$
\Psi_{i j}=\left[\begin{array}{cccc}
\widehat{\Phi}_{i j} & \widehat{E}_{1 i}^{T} & \widehat{E}_{2 i}^{T} & \widehat{E}_{b i j}^{T} \\
* & -\varepsilon_{1 i} I & 0 & 0 \\
* & * & -\varepsilon_{2 i} I & 0 \\
* & * & * & -\varepsilon_{b i j} I
\end{array}\right]<0,
$$


where

$$
\begin{aligned}
& \widehat{\Phi}_{i j}= \\
& {\left[\begin{array}{cccccccc}
\widehat{\Phi}_{11 i} & \bar{\Phi}_{12 i} & \bar{\Phi}_{13} & \bar{\Phi}_{14} & \bar{\Phi}_{15 i j} & \bar{\Phi}_{16} & \bar{\Phi}_{17} & \bar{\Phi}_{18} \\
* & \widehat{\Phi}_{22 i} & t_{3} X A_{2 i}^{T} & t_{4} X A_{2 i}^{T} & \bar{\Phi}_{25 i j} & t_{6} X A_{2 i}^{T} & t_{7} X A_{2 i}^{T} & \bar{\Phi}_{28 i} \\
* & * & \bar{\Phi}_{33 i} & -\bar{P}_{22} & t_{3} B_{i} Y_{j} & 0 & 0 & -t_{3} X^{T} \\
* & * & * & \bar{\Phi}_{44} & t_{4} B_{i} Y_{j} & 0 & 0 & \bar{\Phi}_{48} \\
* & * & * & * & \bar{\Phi}_{55 i j} & t_{6} Y_{j}^{T} B_{i}^{T} & t_{7} Y_{j}^{T} B_{i}^{T} & \bar{\Phi}_{58 i} \\
* & * & * & * & * & \bar{\Phi}_{66} & \bar{\Phi}_{67} & -t_{6} X^{T} \\
* & * & * & * & * & * & \bar{\Phi}_{77} & \bar{\Phi}_{78} \\
* & * & * & * & * & * & * & \bar{\Phi}_{88}
\end{array}\right]<0,} \\
& \widehat{\Phi}_{11 i}=\bar{\Phi}_{11 i}+\varepsilon_{1 i} D_{i} D_{i}^{T}, \\
& \widehat{\Phi}_{22 i}=\bar{\Phi}_{22 i}+\varepsilon_{2 i} D_{i} D_{i}^{T} \text {, } \\
& \widehat{\Phi}_{55 i j}=\bar{\Phi}_{55 i j}+\varepsilon_{b i} D_{i} D_{i}^{T} \text {, }
\end{aligned}
$$

and $\bar{\Phi}$ are the same as in Theorem 4 . Therefore, (41) holds, if and only if the following inequality holds:

$$
\sum_{i=1}^{r} \sum_{j=1}^{r} w_{i}(x(t)) m_{j}(x(t-d(t))) \Psi_{i j}<0 .
$$

To alleviate the conservativeness of our robust stability analysis, similar to the analysis approach of Theorem 4, we introduce $\sum_{i=1}^{r} \sum_{j=1}^{r} w_{i}\left(w_{j}-m_{j}\right) \bar{\Lambda}_{i}=0$ to (43), and the following proof is the same as in Theorem 4 . We can obtain the conditions and (34)-(36). Thus, the fuzzy control system with the time varying state and input delays (34) is robust stable on the basis of the control law $F_{i}=Y_{i} X^{-T}$ under these conditions, and (34)-(36) hold.

Remark 6. If we set $w_{i}(x(t))=m_{j}(x(t)), d_{1}(t)=d_{2}(t)$, the closed-loop system in this paper has the same structure as that of paper [26]. On the other hand, let $w_{i}(x(t))=$ $m_{j}(x(t))$ and $d_{1}(t)=d_{2}(t)=d$; we can obtain the system in [16]. Therefore, the system studied in this paper is more general. Additionally, the information of the membership functions of the fuzzy time delay models and controllers are all considered in the stability analysis. If the fuzzy models and fuzzy controllers share the same fuzzy membership functions, that is, $w_{i}(x(t))=m_{j}(x(t))$, the stability analysis can be referred to $[25,27,28]$. In other words, the problem in [25, 27,28 ] is actually a special case of the one investigated in this paper.

Remark 7. The augmented Lyapunov function with an additional triple-integral term is introduced in analyzing the stability problem for the T-S fuzzy systems with time varying state and input delays. In addition, two integral inequalities are used to derive Theorem 4, and less free-weighting matrices are introduced, which lead to get less conservative results. It can be observed that some existing results are the special cases of this paper. For example, the method in [25] is from (18) with $P_{12}=0, P_{22}=0, Z_{12}=Z_{22}=0, N_{12}=N_{22}=0$, and $R_{1}=R_{2}=0$ in Theorem 4 . The results in [22] are based on system (33) with $d_{2}(t)=0$ and (18) with $M_{2}=0, Z_{12}=$ $Z_{22}=0, Q_{1}=Q_{2}=0$, and $R_{1}=R_{2}=0$.
Remark 8. Different from the general PDC technique, the design method under the imperfect premise matching is much more flexible, because the membership functions of the fuzzy controllers do not need to be chosen the same as those of the fuzzy time delay models. Instead, they can be designed arbitrarily. Thus, the design flexibility is significantly enhanced. On the other hand, some simple membership functions of the fuzzy controllers might be employed, which can reduce the implementation cost.

Remark 9. In [7-11, 15-20], the authors have considered the robust control for uncertain T-S fuzzy systems with state delays. Some delay-dependent conditions and fuzzy controllers are obtained with a traditional Lyapunov-Krasovskii functional method and PDC method, which all are the special cases of Theorem 5 in this paper.

\section{Numerical Examples}

In this section, two numerical examples are given to illustrate the conservativeness and effectiveness of the proposed methods. The first example compares our techniques with the existing ones in the literature for stability analysis, which shows that Theorem 4 in this paper is less conservative than the other results. The second example is used to illustrate the advantage of the robust stability conditions of Theorem 5 and demonstrate how to design robust fuzzy controllers by using our approach.

Example 10. Consider the following fuzzy system with state and input delays:

$$
\begin{gathered}
\dot{x}(t)=\sum_{i=1}^{2} w_{i}(\theta(t))\left[A_{1 i} x(t)+A_{2 i} x\left(t-d_{1}(t)\right)\right. \\
\left.+B_{i} u\left(t-d_{2}(t)\right)\right], \\
A_{11}=\left[\begin{array}{cc}
0 & 0.6 \\
0 & 1
\end{array}\right], \quad A_{21}=\left[\begin{array}{cc}
0.5 & 0.9 \\
0 & 2
\end{array}\right], \\
A_{12}=\left[\begin{array}{ll}
1 & 0 \\
1 & 0
\end{array}\right], \quad A_{22}=\left[\begin{array}{cc}
0.9 & 0 \\
1 & 1.6
\end{array}\right], \\
B_{1}=B_{2}=\left[\begin{array}{l}
1 \\
1
\end{array}\right] .
\end{gathered}
$$

The fuzzy membership functions are selected as [31]

$$
\begin{aligned}
w_{1}\left(x_{1}(t)\right)= & \left(1-\frac{c(t) \sin \left(\left|x_{1}(t)\right|^{-4}\right)^{5}}{1+\exp ^{-100 x_{1}(t)^{3}\left(1-x_{1}(t)\right)}}\right) \\
& \times \frac{\cos \left(x_{1}(t)\right)^{2}}{1+\exp ^{-2.5 x_{1}(t)\left(3+\left(x_{1}(t) / 0.42\right)\right)}} \\
& \times w_{2}\left(x_{1}(t)\right)=1-w_{1}\left(x_{1}(t)\right),
\end{aligned}
$$


TABLE 1: The maximum allowable time delay and feedback gains $\left(\mu_{1}=0, \mu_{2}=0\right)$.

\begin{tabular}{lccc}
\hline Paper & $h_{1}$ & $h_{2}$ & Feedback gains \\
\hline [26] & 0.3120 & 0.3120 & $F_{1}=\left[\begin{array}{ll}1.0598 & -5.6598\end{array}\right]$ \\
& & & $F_{2}=\left[\begin{array}{ll}-1.3068 & -4.1167\end{array}\right]$ \\
Theorem 4 & 0.33 & 0.57 & $F_{1}=\left[\begin{array}{ll}-0.0042 & -0.0449\end{array}\right]$ \\
& & & $F_{2}=\left[\begin{array}{lll}0.0048 & 0.0717\end{array}\right]$ \\
\hline
\end{tabular}

where

$$
\begin{gathered}
x_{1}(t) \in\left[-\frac{\pi}{2} \frac{\pi}{2}\right], \\
c(t)=\frac{\sin \left(x_{1}(t)\right)+1}{40} \in\left[\begin{array}{ll}
-0.05 & 0.05
\end{array}\right] .
\end{gathered}
$$

If we set $d_{1}(t)=d_{2}(t)$ in system (44), we can obtain the system in [26], which implies that our system is more general. Employing the LMIs in [26] and those in Theorem 4 yields the maximum state and input delays $h_{1}$, and $h_{2}$, which guarantee the stability of system (44) and the feedback gains for $\mu_{1}=0$, and $\mu_{2}=0$, when $\rho_{1}=0.75, \rho_{2}=0.95, t_{2}=0.1, t_{3}=$ $0.2, t_{4}=0.7, t_{5}=0.1, t_{6}=0.4, t_{7}=0.1$, and $t_{8}=1.3$ as given in Table 1, which clearly shows the superiority of the results derived in this paper over those obtained from [26].

Example 11. Consider the well-known truck-trailer system, which can be described by the following T-S fuzzy system:

$$
\begin{aligned}
\dot{x}(t)=\sum_{i=1}^{2} w_{i}(\theta(t))[ & \left(A_{1 i}+\Delta A_{1 i}\right) x(t)+A_{2 i} x\left(t-d_{1}(t)\right) \\
& \left.+\left(B_{i}+\Delta B_{i}\right) u\left(t-d_{2}(t)\right)\right], \\
A_{11}= & {\left[\begin{array}{ccc}
-a \frac{v \bar{t}}{L t_{0}} & 0 & 0 \\
a \frac{v \bar{t}}{L t_{0}} & 0 & 0 \\
a \frac{v^{2} \bar{t}^{2}}{2 L t_{0}} & \frac{v \bar{t}}{t_{0}} & 0
\end{array}\right], } \\
A_{21}= & {\left[\begin{array}{lll}
(1-a) \frac{v \bar{t}}{L t_{0}} & 0 & 0 \\
-(1-a) & 0 \\
(1-a) \frac{v^{2} \bar{t}^{2}}{2 L t_{0}} & 0 & 0
\end{array}\right], } \\
B_{1}=\left[\begin{array}{ccc}
\frac{v \bar{t}}{l t_{0}} \\
0 \\
0
\end{array}\right], &
\end{aligned}
$$

$$
\begin{gathered}
A_{12}=\left[\begin{array}{ccc}
-a \frac{v \bar{t}}{L t_{0}} & 0 & 0 \\
a \frac{v \bar{t}}{L t_{0}} & 0 & 0 \\
a \frac{d v^{2} \bar{t}^{2}}{2 L t_{0}} & \frac{d v \bar{t}}{t_{0}} & 0
\end{array}\right], \\
A_{22}=\left[\begin{array}{ccc}
-(1-a) \frac{v \bar{t}}{L t_{0}} & 0 & 0 \\
(1-a) \frac{v \bar{t}}{L t_{0}} & 0 & 0 \\
(1-a) \frac{d v^{2} \bar{t}^{2}}{2 L t_{0}} & 0 & 0
\end{array}\right],
\end{gathered}
$$

$$
B_{2}=\left[\begin{array}{c}
\frac{v \bar{t}}{l t_{0}} \\
0 \\
0
\end{array}\right] \text {, }
$$

where $l=2.8, L=5.5, v=-1.0, \bar{t}=2.0, t_{0}=0.5$, $d=10 t_{0} / \pi, D_{i}=\left[\begin{array}{lll}0.2555 & 0.2555 & 0.2555\end{array}\right]^{T}, E_{1 i}=E_{2 i}=$ $\left[\begin{array}{lll}0.1 & 0 & 0\end{array}\right]^{T}, E_{b i}=\left[\begin{array}{lll}0.1 & 0 & 0\end{array}\right]^{T}(i=1,2)$, and the fuzzy membership functions are selected the same as in Example 10.

(1) Let $a=1$, system (47) is the same as the fuzzy system of [22], which implies that our system is more general. Table 2 gives the maximum input delay value of $h_{2}$, for which the stabilization is guaranteed by Theorem 5 as well as the state feedback gains, when $\rho_{1}=0.75, \rho_{2}=$ $0.95 t_{2}=0.1, t_{3}=0.2, t_{4}=0.7, t_{5}=0.1, t_{6}=$ $0.4, t_{7}=0.8$, and $t_{8}=2$. It is clearly visible that the results derived in this paper are better than those from $[22,28]$.

(2) In the case of $a \neq 1$, the state and input delays of system (47) are all exist. The approach proposed in [22] can not be used to get the maximum of state delay $h_{1}$, as the system in [22] only contains the input delay. However, the method in [28] and Theorem 5 can be used. Here, let $a=0.7, \rho_{1}=0.75, \rho_{2}=0.95, t_{2}=$ $0.1, t_{3}=0.2, t_{4}=0.7, t_{5}=0.1, t_{6}=0.4, t_{7}=$ 0.8 , and $t_{8}=3$, and Table 3 provides the maximum upper bounds of $h_{1}$ and $h_{2}$ and the state feedback gains for which the robust stabilization is guaranteed by Theorem 5 .

By Theorem 5, we can conclude that the uncertain fuzzy model (47) is robust stable based on the following fuzzy control law:

$$
u\left(t-h_{2}\right)=\sum_{j=1}^{2} m_{j}\left(x_{1}\left(t-h_{2}\right)\right) F_{j} x\left(t-h_{2}\right) .
$$

With the PDC design technique, it is required that the fuzzy controller must share the same fuzzy membership functions as those of the fuzzy time delay model. Under such 
TABLE 2: The maximum allowable time delay and feedback gains $\left(\mu_{2}=0\right)$.

\begin{tabular}{lcc}
\hline Paper & $h_{2}$ & Feedback gains \\
\hline$[22]$ & 0.75 & $F_{1}=\left[\begin{array}{lll}3.4227 & -0.3535 & 0.0045\end{array}\right]$ \\
& $F_{2}=\left[\begin{array}{lll}3.5215 & -0.3617 & 0.0056\end{array}\right]$ \\
{$[28]$} & $F_{1}=\left[\begin{array}{lll}3.3219 & -0.2406 & 0.0025\end{array}\right]$ \\
& $F_{2}=\left[\begin{array}{lll}3.3272 & -0.2494 & 0.0026\end{array}\right]$ \\
Theorem 5 & 1.2 & $F_{1}=\left[\begin{array}{lll}-0.0212 & 0.0093 & -0.0024\end{array}\right]$ \\
& & $F_{2}=\left[\begin{array}{lll}-0.0250 & 0.0044 & 0.0014\end{array}\right]$ \\
\hline
\end{tabular}

TABle 3: The maximum allowable time delay and feedback gains $\left(\mu_{1}=\mu_{2}=0\right)$.

\begin{tabular}{lccc}
\hline Paper & $h_{1}$ & $h_{2}$ & Feedback gains \\
\hline [28] & 0.1 & 0.55 & $F_{1}=\left[\begin{array}{llll}3.3219 & -0.2406 & 0.0025\end{array}\right]$ \\
& & & $F_{2}=\left[\begin{array}{llll}3.3272 & -0.2494 & 0.0026\end{array}\right]$ \\
Theorem 5 & 0.4 & \multirow{2}{*}{1.7} & $F_{1}=\left[\begin{array}{lll}-0.0128 & 0.0090 & -0.0017\end{array}\right]$ \\
& & & $F_{2}=\left[\begin{array}{lll}-0.0194 & 0.0012 & 0.0009\end{array}\right]$ \\
\hline
\end{tabular}

a condition, the implementation cost of the fuzzy controller is high by employing these complex membership functions. However, from Theorem 5, the membership functions do not need to be chosen the same as $w_{1}\left(x_{1}(t)\right)$ and $w_{2}\left(x_{1}(t)\right)$. Thus, we can select some simple membership functions instead, such as

$$
\begin{gathered}
m_{1}\left(x_{1}\left(t-h_{2}\right)\right)=0.75 \exp \frac{\left(-x_{1}\left(t-h_{2}\right)-0.38\right)^{2}}{2 \times 0.38^{2}}+0.05 \\
m_{2}\left(x_{1}\left(t-h_{2}\right)\right)=1-m_{1}\left(x_{1}\left(t-h_{2}\right)\right)
\end{gathered}
$$

We also assume that $\rho_{1}=0.75$, and $\rho_{2}=0.95$, such that $m_{j}\left(x_{1}\left(t-h_{2}\right)\right)-\rho_{j} w_{j}\left(x_{1}(t)\right)>0$ for $j=1,2$ and $x_{1}\left(t-h_{2}\right)$. The fuzzy control law $u\left(t-h_{2}\right)=m_{1}\left[\begin{array}{lll}-0.0128 & 0.0090 & -\end{array}\right.$ $0.0017] x+m_{2}\left[\begin{array}{lll}-0.0194 & 0.0012 & 0.0009\end{array}\right] x$ can be employed to stabilize the uncertain fuzzy control systems in (47) with the state and input delays. Figures 1 and 2 illustrate the stable state response and control input under the initial condition of $x(0)=\left[\begin{array}{lll}4 & -1 & 2\end{array}\right]^{T}$.

Remark 12. In Example 11, our method offers less conservative results in the sense of allowing longer time delay and obtaining smaller feedback control gains. Moreover, compared with [22-28], where the fuzzy controller must have the same fuzzy membership functions as those of the fuzzy time delay model, the above membership functions of our fuzzy controller are much simper, which can considerably lower the implementation cost of the fuzzy controller and enhance the design flexibility.

Remark 13. If the function of $c(t)$ in $w_{1}\left(x_{1}(t)\right)$ is unknown, on the basis of the PDC design technique [7-11, 15-20, 2228 ], the fuzzy controller cannot be implemented as $c(t)$ is not available. However, using the proposed design method,

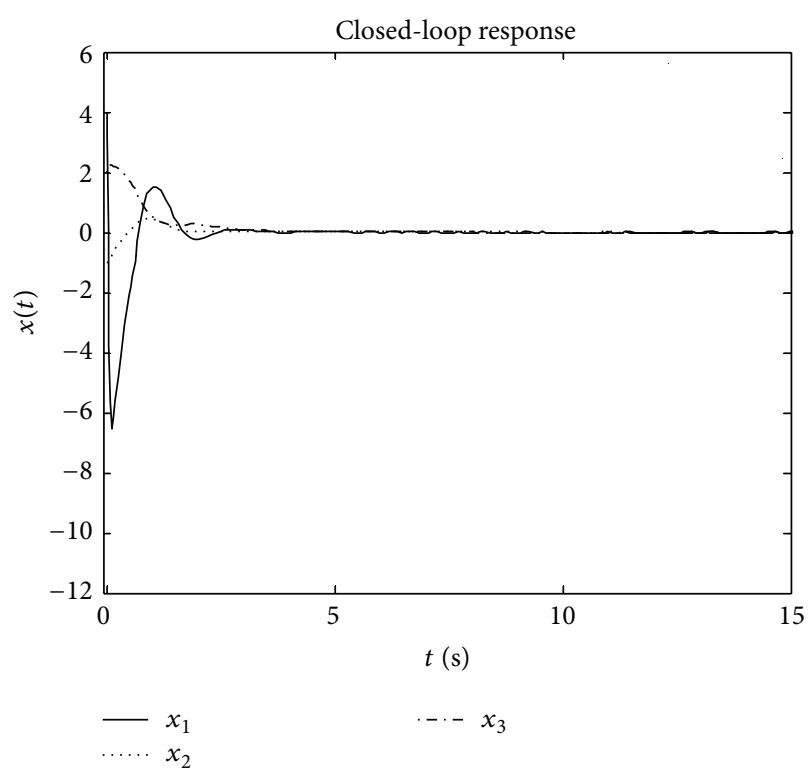

FIgURE 1: The state responses of system (47) with $h_{1}=0.4, h_{2}=1.7$.

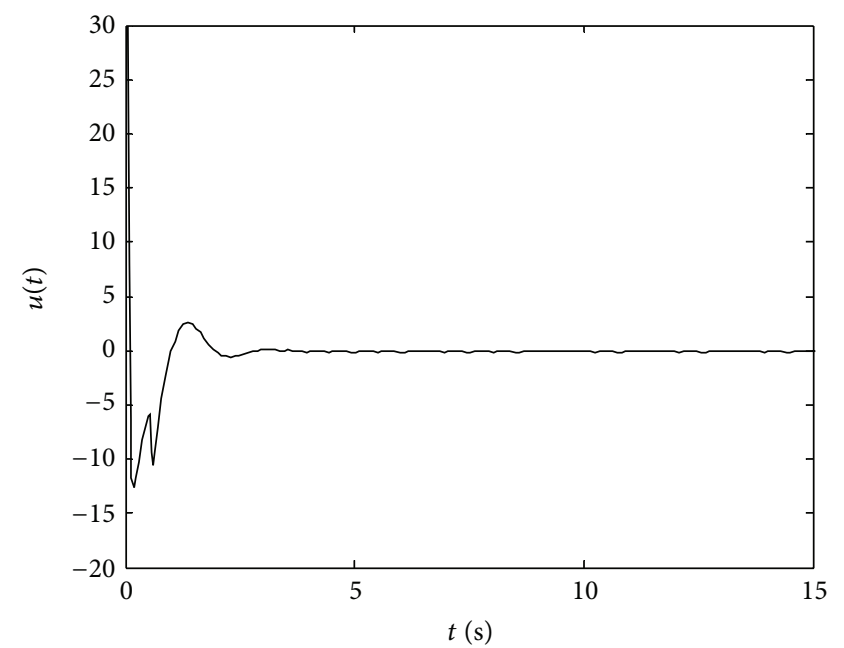

FIGURE 2: The control input of system (47) with $h_{1}=0.4, h_{2}=1.7$.

we can select some simper and well-known membership functions that are different from those of the fuzzy time delay models, for example, $m_{1}\left(x_{1}(t)\right)$ and $m_{2}\left(x_{1}(t)\right)$, so as to realize the fuzzy controller. In other words, the proposed design approach can be applied even for the fuzzy time delay models with uncertain grades of membership.

\section{Conclusions}

In this paper, we study the robust stability and stabilization problems for general nonlinear fuzzy systems with time varying state and input delays. A less conservative delaydependent robust stability criterion has been obtained by introducing a novel augmented Lyapunov function with an additional triple-integral term. Moreover, a new design 
approach different from the traditional PDC design technique is proposed, which can significantly improve the design flexibility and reduce the implementation cost of the fuzzy controller by arbitrarily selecting simple fuzzy membership functions. Two numerical examples are used to illustrate the conservativeness and effectiveness of our proposed methods.

\section{Acknowledgments}

This research work was funded by the NSFC under Grant no. 61273095 and the Academy of Finland under Grants no. 135225 and no. 127299.

\section{References}

[1] T. Takagi and M. Sugeno, "Fuzzy identification of systems and its applications to modeling and control," IEEE Transactions on Systems, Man and Cybernetics, vol. 15, no. 1, pp. 116-132, 1985.

[2] W. H. Ho, S. H. Chen, I. T. Chen, J. H. Chou, and C. C. Shu, "Design of stable and quadratic-optimal static output feedback controllers for T-S-fuzzy-model-based control systems: an integrative computational approach," International Journal of Innovative Computing, Information and Control, vol. 8, no. 1A, pp. 403-418, 2012.

[3] X. J. Su, L. G. Wu, P. Shi, and Y. D. Song, " $H_{\infty}$ model reduction of T-S fuzzy stochastic systems," IEEE Transactions on Systems, Man, and Cybermetics B, vol. 42, no. 6, pp. 1574-1585, 2012.

[4] R. Yang, P. Shi, G.-P. Liu, and H. Gao, "Network-based feedback control for systems with mixed delays based on quantization and dropout compensation," Automatica, vol. 47, no. 12, pp. 2805-2809, 2011.

[5] X. M. Zhang, Z. J. Zhang, and G. P. Lu, "Fault detection for statedelay fuzzy systems subject to random communication delay," International Journal of Innovative Computing, Information and Control, vol. 8, no. 4, pp. 2439-2451, 2012.

[6] F. B. Li and X. Zhang, "Delay-range-dependent robust $H_{\infty}$ filtering for singuar LPV systems with time variant delay," International Journal of Innovative Computing, Information and Control, vol. 9, no. 1, pp. 339-353, 2013.

[7] Y. Y. Cao and P. M. Frank, "Analysis and synthesis of nonlinear time-delay systems via fuzzy control approach," IEEE Transactions on Fuzzy Systems, vol. 8, no. 2, pp. 200-211, 2000.

[8] Y.-Y. Cao and P. M. Frank, "Stability analysis and synthesis of nonlinear time-delay systems via linear Takagi-Sugeno fuzzy models," Fuzzy Sets and Systems, vol. 124, no. 2, pp. 213-229, 2001.

[9] O. M. Kwon and J. H. Park, "Delay-range-dependent stabilization of uncertain dynamic systems with interval time-varying delays," Applied Mathematics and Computation, vol. 208, no. 1, pp. 58-68, 2009.

[10] E. Tian, D. Yue, and Y. Zhang, "Delay-dependent robust $H_{\infty}$ control for T-S fuzzy system with interval time-varying delay," Fuzzy Sets and Systems, vol. 160, no. 12, pp. 1708-1719, 2009.

[11] J. Y. An, T. Li, G. L. Wen, and R. Li, "New stability conditions for uncertain T-S fuzzy systems with interval time-varying delay," International Journal of Control, Automation and Systems, vol. 10, no. 3, pp. 490-497, 2012.

[12] Z. J. Zhang, X. L. Huang, X. J. Ban, and X. Z. Gao, "Stability analysis and controller design of T-S fuzzy systems with timedelay under imperfect premise matching," Journal of Beijing Institute of Technology, vol. 21, no. 3, pp. 387-393, 2012.
[13] O. M. Kwon, M. J. Park, S. M. Lee, and J. H. Park, "Augmented Lyapunov-Krasovskii functional approaches to robust stability criteria for uncertain Takagi-Sugeno fuzzy systems with timevarying delays," Fuzzy Sets and Systems, vol. 201, no. 16, pp. 1-19, 2012.

[14] F. Liu, M. Wu, Y. He, and R. Yokoyama, "New delay-dependent stability criteria for T-S fuzzy systems with time-varying delay," Fuzzy Sets and Systems, vol. 161, no. 15, pp. 2033-2042, 2010.

[15] B. Chen, X. Liu, and S. Tong, "New delay-dependent stabilization conditions of T-S fuzzy systems with constant delay," Fuzzy Sets and Systems, vol. 158, no. 20, pp. 2209-2224, 2007.

[16] J. Yoneyama, "Robust stability and stabilization for uncertain Takagi-Sugeno fuzzy time-delay systems," Fuzzy Sets and Systems, vol. 158, no. 2, pp. 115-134, 2007.

[17] C. Lin, Q.-G. Wang, and T. H. Lee, "Delay-dependent LMI conditions for stability and stabilization of T-S fuzzy systems with bounded time-delay," Fuzzy Sets and Systems, vol. 157, no. 9, pp. 1229-1247, 2006.

[18] X. J. Su, P. Shi, L. G. Wu, and Y. D. Song, "A novel approach to filter design for T-S fuzzy discrete-time systems with timevarying delay," IEEE Transactions on Fuzzy Systems, vol. 20, no. 6, pp. 1114-1129, 2012.

[19] C. Peng and Q.-L. Han, "Delay-range-dependent robust stabilization for uncertain T-S fuzzy control systems with interval time-varying delays," Information Sciences, vol. 181, no. 19, pp. 4287-4299, 2011.

[20] S. T. Li, Y. W. Jing, and X. M. Liu, "Robust control for a class of T-S fuzzy systems with interval time-varying delay," Internatinal Journal of Control, Automation and Systems, vol. 10, no. 4, pp. 737-743, 2012.

[21] X.-M. Zhang, M. Wu, J.-H. She, and Y. He, "Delay-dependent stabilization of linear systems with time-varying state and input delays," Automatica, vol. 41, no. 8, pp. 1405-1412, 2005.

[22] B. Chen, X. Liu, and S. Tong, "Robust fuzzy control of nonlinear systems with input delay," Chaos, Solitons \& Fractals, vol. 37, no. 3, pp. 894-901, 2008.

[23] C. Lin, Q.-G. Wang, and T. H. Lee, "Delay-dependent LMI conditions for stability and stabilization of T-S fuzzy systems with bounded time-delay," Fuzzy Sets and Systems, vol. 157, no. 9, pp. 1229-1247, 2006.

[24] H. J. Lee, J. B. Park, and Y. H. Joo, "Robust control for uncertain Takagi-Sugeno fuzzy systems with time-varying input delay," Journal of Dynamic Systems, Measurement and Control, Transactions of the ASME, vol. 127, no. 2, pp. 302-306, 2005.

[25] C.-H. Lien and K.-W. Yu, "Robust control for Takagi-Sugeno fuzzy systems with time-varying state and input delays," Chaos, Solitons and Fractals, vol. 35, no. 5, pp. 1003-1008, 2008.

[26] Y. K. Su, B. Chen, S. Y. Zhang, and C. Lin, "Delay-dependent stabilization of T-S fuzzy systems with time delay," Control and Decision. Kongzhi yu Juece, vol. 24, no. 6, pp. 921-927, 2009.

[27] B. Chen, X. Liu, C. Lin, and K. Liu, "Robust $H_{\infty}$ control of Takagi-Sugeno fuzzy systems with state and input time delays," Fuzzy Sets and Systems, vol. 160, no. 4, pp. 403-422, 2009.

[28] L. Li and X. Liu, "New results on delay-dependent robust stability criteria of uncertain fuzzy systems with state and input delays," Information Sciences, vol. 179, no. 8, pp. 1134-1148, 2009.

[29] Z. J. Zhang, X. J. Ban, X. L. Huang, and X. Z. Gao, "New delaydependent robust stablity for T-S fuzzy systems with interveval time-varying delay," in Proccedings of the 5th International Joint Conference on Computational Science and Optimization, pp. 826-830, Harbin, China, 2012. 
[30] H. K. Lam and F. H. F. Leung, "LMI-based stability and performance design of fuzzy control systems: fuzzy models and controllers with different premises," in Proceedings of the International Conference on Fuzzy Systerm, pp. 9599-9506, Vancouver, Canada, 2006.

[31] H. K. Lam and M. Narimani, "Stability analysis and perfomance design for fuzzy-model-based control system under imperfect premise matching," IEEE Transactions on Fuzzy Systems, vol. 17, no. 4, pp. 949-961, 2009.

[32] S. Boyd, L. E. Ghaoui, and E. Feron, Linear Matrix Inequality in Systems and Control Theory, SIAM, Philadelphia, Pa, USA, 1994.

[33] I. R. Petersen and C. V. Hollot, "A Riccati equation approach to the stabilization of uncertain linear systems," Automatica, vol. 22, no. 4, pp. 397-411, 1986.

[34] J. Sun, G. P. Liu, and J. Chen, "Delay-dependent stability and stabilization of neutral time-delay systems," International Journal of Robust and Nonlinear Control, vol. 19, no. 12, pp. 1364$1375,2009$. 


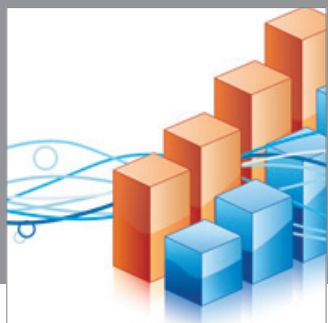

Advances in

Operations Research

mansans

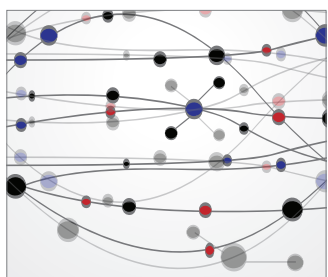

The Scientific World Journal
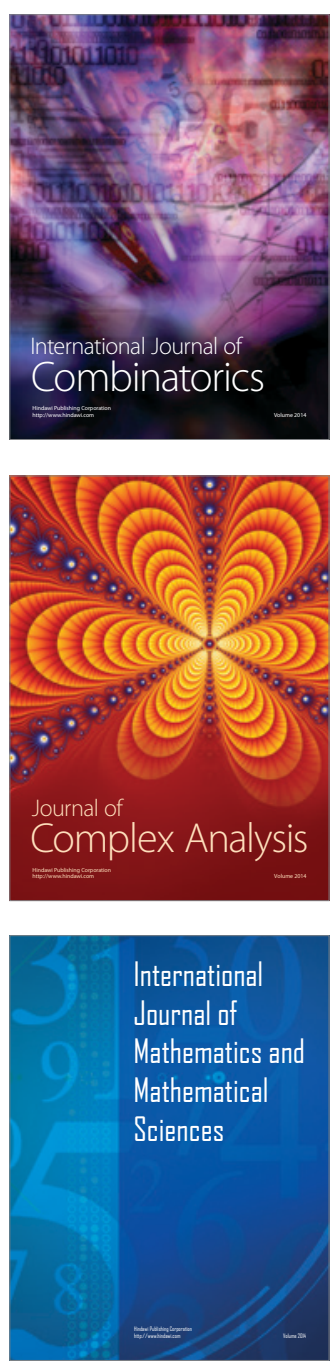
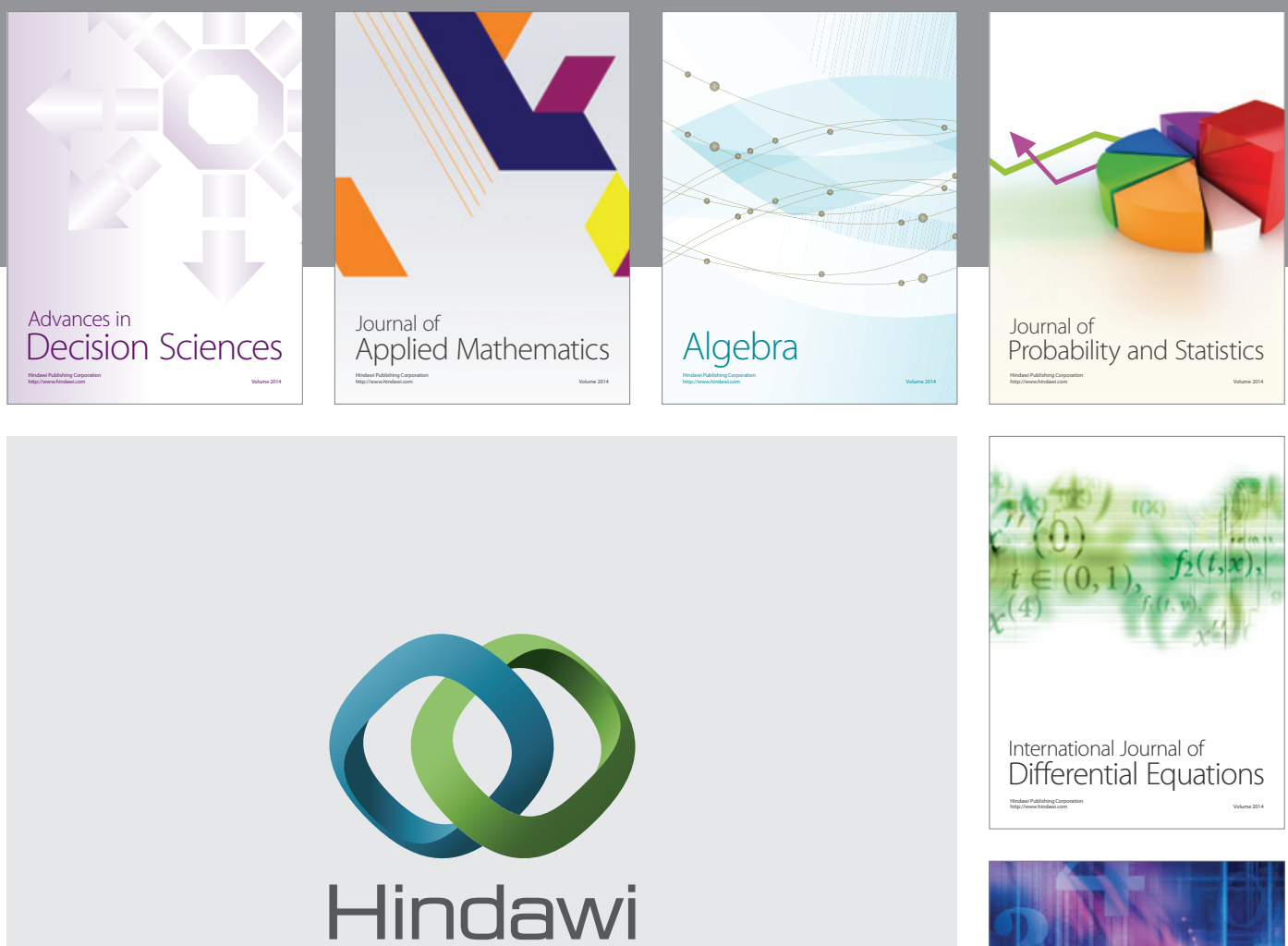

Submit your manuscripts at http://www.hindawi.com
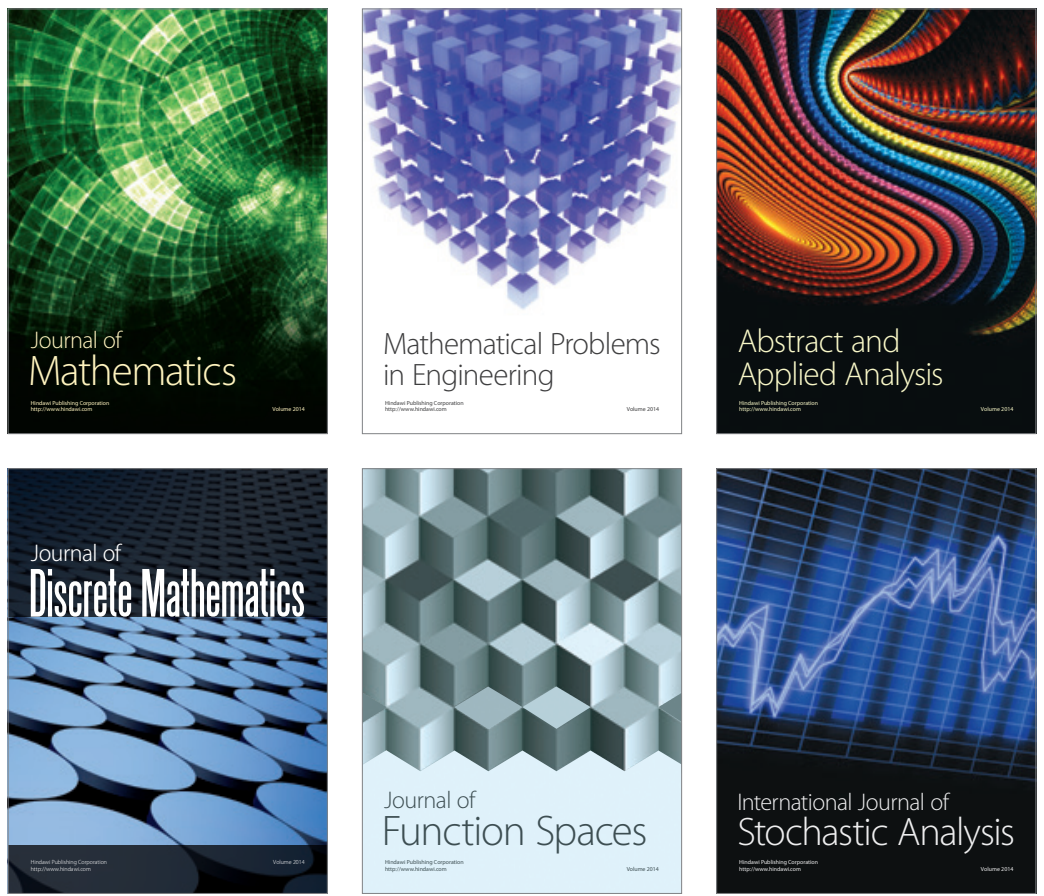

Journal of

Function Spaces

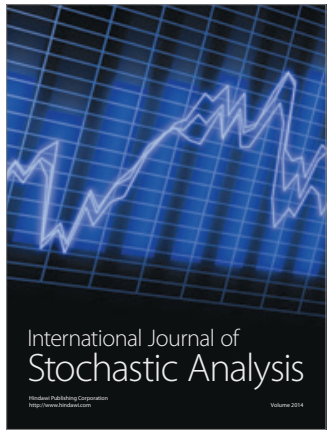

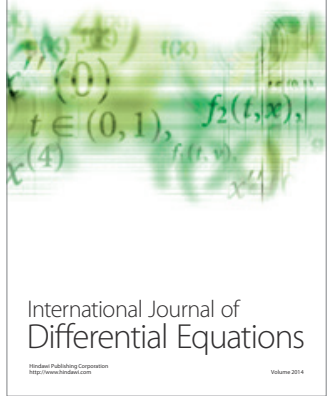
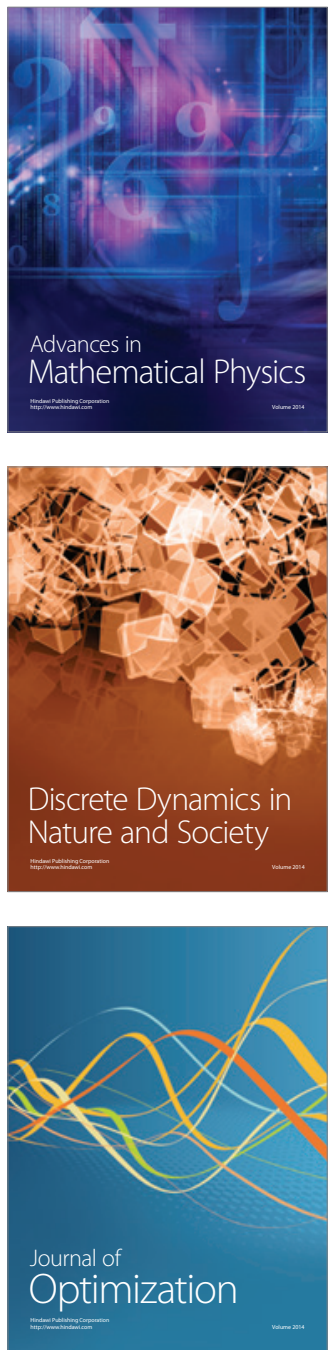